\title{
Experiencia de caries dental en aprendices del programa técnico en salud oral del SENA, Medellín, 2014
}

\author{
Juan Pablo Moreno-Bedoya*, MsC. ${ }_{1}$, Catalina González-Penagos, MsC. ${ }_{1}$, \\ Natalia Calle-Henao, Odon., , Carolina Berruecos-Orozco, Odon.., Melissa \\ Cano-Gómez, Est.
}

\footnotetext{
Facultad de Odontología, Fundación Universitaria Autónoma de las Américas, Medellín, Colombia ${ }_{2}$ Programa técnico en salud oral, Servicio Nacional de Aprendizaje (sENA), Medellín, Colombia
}

Recibido: 24 de mayo del 2015. Aprobado: 30 de junio del 2015.

*Autor de correspondencia: Juan Pablo Moreno-Bedoya. Facultad de Odontología, Fundación Universitaria Autónoma de las Américas, Medellín, Colombia. Calle 34A n. ${ }^{0}$ 76-35. Teléfono: (57) 4 4114444. Correo electrónico: juan.moreno@uam.edu.co

Cómo citar este artículo: Moreno-Bedoya JP, González-Penagos C, Calle-Henao N, Berruecos-Orozco C, Cano-Gómez M. Experiencia de caries dental en aprendices del programa técnico en salud oral del SENA, Medellín, 2014. Rev Nac Odontol. 2015;11(21):65-73. doi: http://dx.doi.org/10.16925/od.v11i21.944

Resumen. Introducción: algunos estudios han abordado con rigurosidad la asociación entre las patologías orales más prevalentes y el nivel socioeconómico y educativo, y han encontrado que cuanto más bajo el nivel socioeconómico y educativo, la prevalencia de las patologías orales aumenta. El propósito de este estudio fue describir las variables sociodemográficas, los hábitos de salud oral y la experiencia y prevalencia de caries dental de los aprendices del programa técnico en salud oral del senA, en Medellín, Colombia. Materiales y métodos: estudio descriptivo transversal, en 90 aprendices con edades entre 15 y 40 años. Previamente, se calibraron dos odontólogas, se hizo la evaluación de los estudiantes, y se calcularon las proporciones de experiencia y prevalencia de caries dental haciendo uso del índice COP-D recomendado por la oMs. Se hizo la descripción de las variables, y se observó la asociación entre la variable dependiente y las variables sociodemográficas, los hábitos de higiene oral y el acceso a los servicios de salud. Resultados: el 65,6\% (59 aprendices) pertenecía a un estrato socioeconómico bajo. El 61,1\% (55 aprendices) estaba afiliado al régimen subsidiado de salud. La prevalencia de caries dental fue del 30\%, la experiencia fue de 67,7\%. Hubo asociación estadísticamente significativa al relacionar “QQué elementos usa para lavarse los dientes?” con la variable dependiente $(\mathrm{p}=0,037)$. Conclusiones: en general la salud dental de la población fue buena, lo que coincide con la progresiva disminución de la prevalencia de caries dental evidenciada en el tercer y cuarto Estudio Nacional de Salud Bucal en población del mismo grupo etario.

Palabras clave: adulto, caries dental, educación técnica dental. 


\title{
Experiences on Tooth Decay in Trainees of SENA Oral Health Technical Program, Medellín, 2014
}

\begin{abstract}
Introduction: some studies have strictly treated the association between the most frequent oral pathologies and the social economic and educational levels, finding out that the lower the social economic and educative level, the higher the presence of oral pathologies. The goal of this study was to describe the social demographic variables, oral health habits, experience and prevalence of tooth decay of the trainees of SENA oral health technical program, in Medellín, Colombia. Materials and methods: descriptive cross sectional study in 90 trainees from 15 to 40 years of age. Two dental surgeons were previously calibrated, students were evaluated, and the experiences and prevalence proportions on tooth decay were calculated by the COP-D Index, recommended by the wHO. The description of the variables was made, the association between the dependent variable and the social demographic variables was observed, as well as oral hygiene habits and access to health services. Results: $65.6 \%$ (59 trainees) belonged to a low social class. $61.1 \%$ (55 trainees) were affiliated to the subsidized health system. Prevalence of tooth decay was $30 \%$, experience was $67.7 \%$. There was a significant statistic association between "What elements do you use to brush your teeth?" with the dependent variable $(\mathrm{p}=0.037)$. Conclusions: in general the population's oral health was good, which coincides with the progressive decrease of prevalence of tooth decay showed in the third and fourth national study on oral health, in the same age range.
\end{abstract}

Keywords: adult, tooth decay, dental technical education.

\section{Experiência de cárie dentária em aprendizes do programa técnico em saúde do SENA, Medellín, 2014}

Resumo. Introdução: alguns estudos têm tratado rigorosamente a associação entre as patologias orais mais prevalentes e o nível socioeconómico e educativo, e tem achado que quanto mais baixo o nível socioeconómico e educacional, a prevalência das patologias orais aumenta. O escopo deste estudo foi descrever as variáveis sócio-demográficas, os hábitos de saúde oral, a experiência e prevalência de cárie dentária dos aprendizes do programa técnico em saúde oral do SENA, em Medellín, Colômbia. Materiais e métodos: estudo descritivo transversal, em 90 aprendizes, com idades entre os 15 e 40 anos. Previamente duas odontologistas foram calibradas, fez-se a avaliação dos estudantes, calcularamse as proporções de experiência e prevalência de cárie dentária usando o índice COP-D recomendado pela oms. Foi feita a descrição das variáveis, foi revisitada a associação entre a variável dependente e as variáveis sócio-demográficas, os hábitos de higiene oral e o acesso aos serviços de saúde. Resultados: 65,6\% (59 aprendizes) pertencia a um nível socioeconómico baixo. 61,1\% (55 aprendizes) estava afiliado ao regime subsidiado de saúde. A prevalência de cárie dentária foi de $30 \%$, a experiência foi de $67,7 \%$. Houve associação estatisticamente significativa relacionando "Quais elementos usa para se escovar os dentes?” Com a variável dependente $(\mathrm{p}=0,037)$. Conclusões: em geral a saúde dentaria da população foi boa, coincidindo com a progressiva diminuição da prevalência de cárie dentária evidenciada no terceiro e quarto estudo nacional de saúde bucal em população do mesmo grupo etário.

Palavras-chave: adulto, caries dental, educação técnica dental. 


\section{Introducción}

En el 2007, la Organización Mundial de la Salud (oMs) informó sobre la caries dental como una de las principales causas de morbilidad bucal, con una prevalencia entre el $60 \%$ y $80 \%$ de la población mundial, que afecta en su mayoría a grupos vulnerables debido a sus condiciones biológicas, psicológicas, sociales y económicas [1]. Esta es una enfermedad multifactorial asociada con la interrelación de varios factores como los azúcares en la dieta, las bacterias de la boca, la existencia de dientes susceptibles o predispuestos, y el tiempo, lo que permite esclarecer de una forma más precisa la formación. Asimismo, la falta de higiene bucal, los niveles bajos de educación y la precariedad en las condiciones de vida son factores que contribuyen a su aparición [2], [3].

Los efectos nocivos de la caries tienen que ver con la afectación de funciones básicas vitales como la alimentación y de otras más complejas que influyen psicosocialmente en la interacción con otras personas. Esto permite afirmar que tanto la calidad de vida como la salud integral de las personas se ven comprometidas como consecuencia de la enfermedad [4].

En Colombia, según el III Estudio Nacional de Salud Bucal (eNSAB III), realizado en 1998, la prevalencia de caries dental fue del $65,3 \%$ para la población general, con un COP-D de 10 , siendo la prevalencia mayor en los hombres $(67,8 \%)$ que en las mujeres (63\%). El grupo de edad comprendido entre 15 y 19 años es el más afectado, con una prevalencia de $89 \%$, y de $75 \%$ en la población adulta joven (20-34 años) [5].

$\mathrm{Al}$ mismo tiempo, el estudio reportó que -en relación con el comportamiento de la demanda de servicios de la población- los residentes de la zona urbana y los de mayor nivel socioeconómico o escolaridad presentan más interés por mantener conductas preventivas, lo cual está asociado, posiblemente, con diversos condicionamientos sociales de estética y mayor acceso a consultas, principalmente en instituciones privadas. También, agrega algunas diferencias positivas en los hábitos de higiene oral de la población colombiana con respecto al segundo estudio de morbilidad oral (19771980). Para 1998, el 98\% de la población reportó que usa el cepillo y la crema dental, y el $37,4 \%$ usa la seda dental de manera rutinaria, en comparación con el $31,5 \%$ encontrado en 1977-80 [6].
Numerosos estudios han señalado el efecto negativo que tienen las desigualdades socioeconómicas sobre distintos aspectos relacionados con la salud: a medida que desciende la posición socioeconómica, son peores los indicadores de salud. El caso de la salud bucal es similar: si se tienen en cuenta distintos indicadores de posición socioeconómica durante los últimos años en países latinoamericanos como Brasil y México, se observa que ha incrementado el número de estudios en los que se demuestran las desigualdades socioeconómicas que existen tanto en los indicadores de salud bucal como en la cobertura y utilización de los servicios odontológi$\cos$ [7], [8].

Colombia es el país número 14 en cuanto a mayor desigualdad entre los 134 observados por el Programa de Naciones Unidas para el Desarrollo (PNUD), lo que muestra que la desigualdad entre el 2012 y el 2013 se mantuvo prácticamente igual. En esos años, el coeficiente de Gini fue de 0,539, y en el 2014, de 0,538 [9], [10].

Una de las instituciones que más trabaja para contribuir en la mejoría de las condiciones de los colombianos es el Servicio Nacional de Aprendizaje (SENA), que apuesta por la inclusión social en articulación con la política nacional: más empleo y menos pobreza. El SENA es un establecimiento público del orden nacional adscrito al Ministerio del Trabajo de Colombia, que ofrece formación gratuita a los colombianos con programas técnicos, tecnológicos y complementarios, con el fin de que sus egresados entren a engrosar las actividades productivas de las empresas y de la industria, para obtener mejor competitividad y producción con los mercados globalizados [11], [12].

Este estudio se realizó con el objetivo de describir las variables sociodemográficas, los hábitos de salud oral, y la experiencia y prevalencia de caries dental en los aprendices del programa técnico en salud oral de esta institución en el segundo semestre del 2014.

\section{Métodos}

Se hizo un estudio descriptivo con enfoque empírico-analítico, transversal, en una muestra inicial de 100 aprendices del programa técnico en salud oral del sena Medellín, entre los cuales 90 decidieron participar, siendo esta la población final. Se aplicó la encuesta de salud bucodental avalada por 
la oms [13], que contenía información de las variables sociodemográficas, los conocimientos, hábitos y servicios odontológicos, y el acceso y la evaluación clínica. Dicho instrumento fue aplicado por el grupo de investigadores y fue probado mediante prueba piloto en un grupo de 10 personas, lo cual permitió evaluar la consistencia interna y la comprensión de las preguntas.

Para la aplicación del instrumento, fueron entrenadas y calibradas dos de las investigadoras odontólogas en la detección de caries dental, con un índice Kappa ínterexaminador de 0,85. Los exámenes se realizaron en las instalaciones del SENA del municipio de Copacabana, en un espacio abierto, ventilado, iluminado, con luz natural y en donde se utilizó una lámpara de luz blanca como fuente de iluminación directa.

Se utilizó el índice de dientes cariados, perdidos y obturados (CPOD), recomendado por la oMs (1997) como el indicador de elección para los relevamientos epidemiológicos poblacionales para medir historia y presencia de la enfermedad y sus secuelas. Se utilizó el criterio de lesión cavitada propuesto por la oms.

Después de la limpieza dental y de haber secado los dientes, se valoraron las superficies dentales mediante inspección visual con la ayuda de un espejo bucal plano; los resultados del examen fueron consignados por un anotador en el formato antes mencionado.

Las técnicas de procesamiento y análisis de datos contemplaron la descripción de las variables sociodemográficas, de acuerdo con los objetivos específicos planteados, además de la forma de representación y las técnicas utilizadas para la medición y el análisis. Con base en esto, el estudio se desarrolló en dos fases: en la primera, se hizo el análisis univariado, en el cual se analizaron las variables sociodemográficas. Después se hizo el análisis bivariado, que consistió en establecer los factores sociodemográficos asociados con la experiencia de caries de los aprendices del programa técnico profesional en salud oral del SENA. Las técnicas utilizadas para la medición y el análisis fueron las razones de prevalencia con sus respectivos intervalos de confianza del 95\% y la prueba chi cuadrado; para las variables cuantitativas que no tenían distribución normal, como la edad, se aplicó la U-Mann Withney.

Para el procesamiento de la información, se elaboró una base de datos en Excel, luego se digitalizó y procesó en el software Prisma con licencia de la Fundación Universitaria Autónoma de las Américas.

Se estimaron proporciones para la prevalencia y la experiencia de caries, y se calcularon los promedios del índice COPD. Se exploraron diferencias en los índices de caries según edad, sexo, estrato socioeconómico, nivel de escolaridad y afiliación al sistema general de seguridad social en salud, por medio de pruebas no paramétricas, dado que no tenían una distribución normal por la gran variabilidad en el grupo de estudio, para lo cual se utilizó un nivel de significación de $5 \%$.

La presente investigación cumplió con los requisitos éticos de la investigación en salud de la Resolución 8430 de 1993, emanada por el Ministerio de Salud y Protección Social del Estado colombiano, según la cual esta investigación se clasifica como una investigación con riesgo mínimo [14]. Los participantes fueron informados previamente de los objetivos de la investigación, de sus derechos, así como del procedimiento y el tiempo en que se llevaría a cabo.

\section{Resultados}

De las características sociodemográficas de la población, se tiene que la edad mínima reportada fue de 15 años y la máxima fue de 40 años. La edad promedio de los aprendices encuestados es de 24,44 años, siendo los 17 años la edad más frecuente. Seis de cada 10 aprendices pertenecen a un estrato socioeconómico bajo (1-2). Tres quintas partes de los aprendices pertenecen al régimen subsidiado. Respecto al nivel de escolaridad de los encuestados, se encontró que el $94,4 \%$ (85) había cursado la secundaria completa o tenía estudios técnico-superiores. Se halló una prevalencia de caries dental del $30 \%$ en una población que en su mayoría fue femenina, la experiencia de caries dental fue del $67,7 \%$ y se halló un promedio de menos de un diente cariado por individuo $(0,42)$. En lo que respecta a dientes obturados y perdidos por esta misma razón, el promedio fue de 1,46 y 0,5 , respectivamente; el cop comunitario hallado fue de 0,67 .

En cuanto a la experiencia de caries dental, 59 de 87 mujeres habían tenido evidencia de esta, las 28 restantes estaban sanas al momento de la revisión. En la tabla 1 se muestran las características sociodemográficas de la población de estudio y su relación con la experiencia de caries dental. 
Tabla 1. Distribución porcentual de la experiencia de caries dental según estado civil, estrato, procedencia, ocupación y tipología familiar, en aprendices del programa técnico en salud oral del sENA, Medellín, 2014

\begin{tabular}{|c|c|c|c|c|c|}
\hline \multirow{2}{*}{\multicolumn{2}{|c|}{ Îndice COP }} & \multicolumn{2}{|c|}{ Sin experiencia de caries } & \multicolumn{2}{|c|}{ Con experiencia de caries } \\
\hline & & \multirow{2}{*}{$\begin{array}{l}\mathrm{N} \\
24 \\
\end{array}$} & \multirow{2}{*}{$\begin{array}{c}\% \\
34,29 \\
\end{array}$} & \multirow{2}{*}{$\begin{array}{l}\mathrm{N} \\
46 \\
\end{array}$} & \multirow{2}{*}{$\begin{array}{c}\% \\
65,71 \\
\end{array}$} \\
\hline \multirow{4}{*}{ Estado civil } & Soltero & & & & \\
\hline & Casado & 2 & 18,18 & 9 & 81,82 \\
\hline & Otro & 3 & 33,33 & 6 & 66,67 \\
\hline & Total & 29 & 32,22 & 61 & 67,78 \\
\hline \multirow{4}{*}{ Estrato socioeconómico } & Bajo & 19 & 32,20 & 40 & 67,8 \\
\hline & Medio & 10 & 35,71 & 18 & 64,29 \\
\hline & Otro & 0 & 0,00 & 3 & 100,00 \\
\hline & Total & 29 & 32,22 & 61 & 67,78 \\
\hline \multirow{3}{*}{ Procedencia } & Medellín & 19 & 35,85 & 34 & 64,15 \\
\hline & Otro municipio & 10 & 27,03 & 27 & 72,97 \\
\hline & Total & 29 & 32,22 & 61 & 67,78 \\
\hline \multirow{3}{*}{ Ocupación } & Estudia exclusivamente & 26 & 31,33 & 57 & 68,67 \\
\hline & Trabaja y estudia & 3 & 42,86 & 4 & 57,14 \\
\hline & Total & 29 & 32,22 & 61 & 67,78 \\
\hline \multirow{5}{*}{ Tipología familiar } & Nuclear & 8 & 27,59 & 21 & 72,41 \\
\hline & Monoparental & 8 & 34,78 & 15 & 65,22 \\
\hline & \begin{tabular}{|l} 
Extendida \\
\end{tabular} & 11 & 33,33 & 22 & 66,67 \\
\hline & Otro & 2 & 40,00 & 3 & 60,00 \\
\hline & Total & 29 & 32,22 & 61 & 67,78 \\
\hline
\end{tabular}

Fuente: elaboración propia

La proporción de experiencia de caries dental fue superior en todos los grupos etarios. El COP comunitario fue de 0,67 ; sin embargo, se observó un incremento a medida que aumentaba elgrupo etario, lo cual se evidenció al observar que el promedio del índice cop en el grupo de edad de 15 a 19 años fue de 0,8 ; en los grupos de 20 a 29 y de 30 a 40 años fue de 1,1 y 5,0 , respectivamente. No obstante, no se presentó asociación estadísticamente significativa con esta variable y la edad (figura 1).

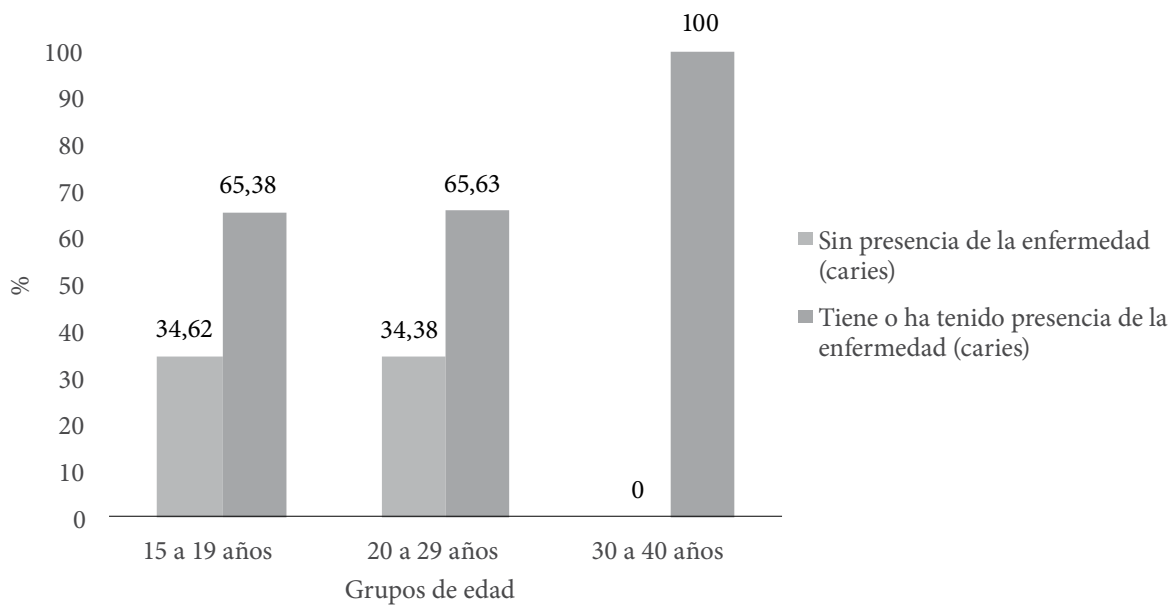

Figura 1. Distribución porcentual de la experiencia de caries dental según grupos de edad, en aprendices del programa técnico en salud oral del SENA, Medellín, 2014

Fuente: elaboración propia 
Frente a la etiología de la caries dental, el $70,89 \%$ (56) de los aprendices que han tenido experiencia de caries dental consideró que esta se produce por tener un mal cepillado, a diferencia del $50 \%$ (4) de los que también tenían experiencia de caries, que lo atribuyó al hecho de una ingesta elevada de dulces.
En lo que respecta a los hábitos de higiene oral y a la experiencia de caries dental, se halló que el $56,52 \%$ de los que decían cepillar sus dientes dos veces al día ha tenido experiencia de caries, y el $43,48 \%$ con este mismo hábito estaba sano. En la tabla 2, se muestran los hábitos de higiene oral de la población de estudio y su relación con la experiencia de caries dental.

Tabla 2. Distribución porcentual de la experiencia de caries dental según hábitos de higiene oral (cuántas veces al día se cepilla, usa la seda dental, cuántas veces al día usa la seda dental, usa enjuague bucal, usa crema dental), en aprendices del programa técnico en salud oral del SENA, Medellín, 2014

\begin{tabular}{|c|c|c|c|c|c|c|c|}
\hline \multirow{2}{*}{\multicolumn{2}{|c|}{ Índice (COP) }} & \multicolumn{2}{|c|}{ Sin experiencia de caries } & \multicolumn{2}{|c|}{ Con experiencia de caries } & \multicolumn{2}{|c|}{ Total } \\
\hline & & $\mathbf{N}$ & $\%$ & $\mathrm{~N}$ & $\%$ & $\mathrm{~N}$ & $\%$ \\
\hline \multirow{4}{*}{ ¿Cuántas veces al día se cepilla? } & Una sola vez al día & 0 & 0,00 & 2 & 100,00 & 2 & 100,00 \\
\hline & Dos veces al día & 10 & 43,48 & 13 & 56,52 & 23 & 100,00 \\
\hline & Más de tres veces al día & 19 & 29,23 & 46 & 70,77 & 65 & 100,00 \\
\hline & Total & 29 & 32,22 & 61 & 67,78 & 90 & 100,00 \\
\hline \multirow{3}{*}{ ¿Usa la seda dental? } & Sí & 14 & 31,82 & 30 & 68,18 & 44 & 100,00 \\
\hline & No & 15 & 32,61 & 31 & 67,39 & 46 & 100,00 \\
\hline & Total & 29 & 32,22 & 61 & 67,78 & 90 & 100,00 \\
\hline \multirow{5}{*}{$\begin{array}{l}\text { ¿Cuántas veces al día usa la seda } \\
\text { dental? }\end{array}$} & Una vez al día & 7 & 31,82 & 15 & 68,18 & 22 & 100,00 \\
\hline & Dos veces al día & 2 & 14,29 & 12 & 85,71 & 14 & 100,00 \\
\hline & Tres veces al día & 5 & 62,50 & 3 & 37,50 & 8 & 100,00 \\
\hline & Ninguna vez & 0 & 0,00 & 0 & 0,00 & 0 & 0,00 \\
\hline & Total & 14 & 31,82 & 30 & 68,18 & 44 & 100,00 \\
\hline \multirow{3}{*}{ ¿Usa enjuague bucal? } & Sí & 9 & 25,71 & 26 & 74,29 & 35 & 100,00 \\
\hline & No & 20 & 36,36 & 35 & 63,64 & 55 & 100,00 \\
\hline & Total & 29 & 32,22 & 61 & 67,78 & 90 & 100,00 \\
\hline \multirow{3}{*}{ ¿Usa crema dental? } & Sí & 29 & 32,22 & 61 & 67,78 & 90 & 100,00 \\
\hline & No & 0 & 0,00 & 0 & 0,00 & 0 & 0,00 \\
\hline & Total & 29 & 32,22 & 61 & 67,78 & 90 & 100,00 \\
\hline
\end{tabular}

Fuente: elaboración propia

$\mathrm{Al}$ asociar este índice con las variables que dan respuesta a los conocimientos, las prácticas y los hábitos de las personas encuestadas, existe una asociación significativa entre la variable "Qué elementos usa para la lavarse los dientes" $(\mathrm{P}<0,05)$ y el índice, lo cual indica que existen diferencias entre quienes usan cepillo dental, cepillo y crema dental, cepillo, crema dental, seda dental, cepillo, crema dental, seda dental y enjuague bucal, dedos de la mano, enjuague con agua, y no usa ningún elemento. Ninguna de las otras variables relacionadas con este aspecto tuvo asociación con el cop (tabla 3).

La relación entre los elementos usados en la higiene oral y el índice cop está dada posiblemente por la efectividad que obtiene cada elemento de higiene oral al adicionarse a otro que complementa su función, lo cual genera ventaja en algún nivel, en comparación con quienes usan el mínimo de elementos para este hábito (tabla 3). 
Tabla 3. Distribución porcentual de la experiencia de caries dental según variables relacionadas con hábitos de higiene, en aprendices del programa técnico en salud oral del sENA, Medellín, 2014

\begin{tabular}{|c|c|c|c|c|c|c|c|c|c|}
\hline \multirow{3}{*}{\multicolumn{2}{|c|}{ Hábitos de higiene }} & \multicolumn{8}{|c|}{ Índice (COP) } \\
\hline & & \multicolumn{2}{|c|}{$\begin{array}{l}\text { Sin presencia de } \\
\text { la enfermedad } \\
\text { (caries) }\end{array}$} & \multicolumn{2}{|c|}{$\begin{array}{l}\text { Tiene o ha tenido } \\
\text { presencia de } \\
\text { la enfermedad } \\
\text { (caries) }\end{array}$} & \multicolumn{2}{|c|}{ Total } & \multirow[t]{2}{*}{$\begin{array}{l}\text { Chi cua- } \\
\text { drado }\end{array}$} & \multirow[t]{2}{*}{$\begin{array}{l}\text { Valor p } \\
(<0,05)\end{array}$} \\
\hline & & $\mathrm{N}$ & $\%$ & $\mathrm{~N}$ & $\%$ & $\mathrm{~N}$ & $\%$ & & \\
\hline \multirow{8}{*}{$\begin{array}{l}\text { ¿Qué elementos } \\
\text { usa para lavarse los } \\
\text { dientes? }\end{array}$} & Cepillo dental & 0 & 0,00 & 0 & 0,00 & 0 & 0,00 & \multirow{8}{*}{6,599} & \multirow{8}{*}{0,037} \\
\hline & Cepillo y crema dental & 11 & 37,93 & 22 & 36,07 & 33 & 36,67 & & \\
\hline & $\begin{array}{l}\text { Cepillo, crema dental, seda } \\
\text { dental }\end{array}$ & 16 & 55,17 & 21 & 34,43 & 37 & 41,11 & & \\
\hline & $\begin{array}{l}\text { Cepillo, crema dental, seda } \\
\text { dental, enjuague bucal }\end{array}$ & 2 & 6,90 & 18 & 29,51 & 20 & 22,22 & & \\
\hline & Dedos de la mano & 0 & 0,00 & 0 & 0,00 & 0 & 0,00 & & \\
\hline & Enjuague con agua & 0 & 0,00 & 0 & 0,00 & 0 & 0,00 & & \\
\hline & No usa nada & 0 & 0,00 & 0 & 0,00 & 0 & 0,00 & & \\
\hline & Total & 29 & 100,00 & 61 & 100,00 & 90 & 100,00 & & \\
\hline \multirow{4}{*}{$\begin{array}{l}\text { ¿Cuántas veces al día } \\
\text { se cepilla los dientes? }\end{array}$} & Una vez al día & 0 & 0,00 & 2 & 3,28 & 2 & 2,22 & \multirow{4}{*}{2,551} & \multirow{4}{*}{0,279} \\
\hline & Dos veces al día & 10 & 34,48 & 13 & 21,31 & 23 & 25,56 & & \\
\hline & Más de tres veces al día & 19 & 65,52 & 46 & 75,41 & 65 & 72,22 & & \\
\hline & Total & 29 & 100,00 & 61 & 100,00 & 90 & 100,00 & & \\
\hline \multirow{6}{*}{$\begin{array}{l}\text { ¿Cada cuánto cambia } \\
\text { de cepillo de dientes? }\end{array}$} & De 0 a 3 meses & 22 & 75,86 & 45 & 73,77 & 67 & 74,44 & \multirow{6}{*}{0,058} & \multirow{6}{*}{0,971} \\
\hline & De 3 a 6 meses & 6 & 20,69 & 14 & 22,95 & 20 & 22,22 & & \\
\hline & De 6 a 9 meses & 1 & 3,45 & 2 & 3,28 & 3 & 3,33 & & \\
\hline & Más de 9 meses & 0 & 0,00 & 0 & 0,00 & 0 & 0,00 & & \\
\hline & No lo cambia & 0 & 0,00 & 0 & 0,00 & 0 & 0,00 & & \\
\hline & Total & 29 & 100,00 & 61 & 100,00 & 90 & 100,00 & & \\
\hline \multirow{3}{*}{ ¿Usa seda dental? } & Sí & 14 & 48,28 & 30 & 49,18 & 44 & 48,89 & \multirow{3}{*}{0,006} & \multirow{3}{*}{0,936} \\
\hline & No & 15 & 51,72 & 31 & 50,82 & 46 & 51,11 & & \\
\hline & Total & 29 & 100,00 & 61 & 100,00 & 90 & 100,00 & & \\
\hline \multirow{5}{*}{$\begin{array}{l}\text { ¿Cuántas veces al día } \\
\text { usa seda dental? }\end{array}$} & Una vez al día & 7 & 24,14 & 15 & 24,59 & 22 & 24,44 & \multirow{5}{*}{5,425} & \multirow{5}{*}{0,143} \\
\hline & Dos veces al día & 2 & 6,90 & 12 & 19,67 & 14 & 15,56 & & \\
\hline & Tres veces al día & 5 & 17,24 & 3 & 4,92 & 8 & 8,89 & & \\
\hline & Ninguna vez & 15 & 51,72 & 31 & 50,82 & 46 & 51,11 & & \\
\hline & Total & 29 & 100,00 & 61 & 100,00 & 90 & 100,00 & & \\
\hline \multirow{3}{*}{ ¿Usa enjuague bucal? } & Sí & 9 & 31,03 & 26 & 42,62 & 35 & 38,89 & \multirow{3}{*}{1,111} & \multirow{3}{*}{0,292} \\
\hline & No & 20 & 68,97 & 35 & 57,38 & 55 & 61,11 & & \\
\hline & Total & 29 & 100,00 & 61 & 100,00 & 90 & 100,00 & & \\
\hline
\end{tabular}

Fuente: elaboración propia

\section{Discusión}

Si bien los estudios de prevalencia y experiencia de caries dental son abundantes [15]-[17], trabajos enfocados específicamente en personal auxiliar en formación no lo son; por tanto, los estudios referenciados abordan la comparación con higienistas dentales, auxiliares de odontología y personal en formación profesional. Al comparar el presente estudio con una investigación sobre las tendencias actuales en el personal auxiliar de odontología en Andalucía, España, se observan semejanzas en factores demográficos como sexo, edad, estado civil, nivel de escolaridad, estrato socioeconómico, y situación laboral y académica.

En lo concerniente al estrato socioeconómico, en el mismo estudio se encontró que el $82 \%$ de la población pertenecía a un nivel económico medio, diferente de lo encontrado en el estudio actual, en el que el 65,6\% de los aprendices pertenecía a un nivel socioeconómico bajo [18].

$\mathrm{Al}$ analizar los resultados obtenidos en esta investigación - sobre los conocimientos, las prácticas y los hábitos- y compararlos con un estudio 
realizado sobre creencias de caries e higiene oral en adolescentes del Valle del Cauca (Colombia), se encontraron analogías con respecto a la etiología de la caries dental: en dicho estudio el 65\% de los adolescentes encuestados cree que es consecuencia de la mala higiene oral y el $7 \%$ considera que es por el consumo de azúcares [3].

En relación con la frecuencia del cepillado, en el estudio antes mencionado se observó que el 79\% de las personas estudiadas se cepillaba tres o más veces al día, y el 55\% refería hacer uso de la seda dental, en comparación con el presente estudio, en el que el $72,2 \%$ se cepilla los dientes con la misma frecuencia (tres o más veces) y el $48,9 \%$ usa la seda dental como parte de su rutina diaria [3].

Al comparar la prevalencia de caries dental de los resultados del último Estudio Nacional de Salud Bucal ENSAB IV (2015) [18] —específicamente el grupo etario entre 20 y 34 años - con el presente estudio (y el promedio de edad de los aprendices valorados - 24,4 años), se observa una diferencia en la prevalencia de la enfermedad, siendo mayor en el ENSAB, con un reporte de $52,81 \%$ en comparación con el $30 \%$ de los aprendices del sENA. Esta diferencia puede ser entendible considerando que la población estudiada en el sENA se enfocó en jóvenes que contaban con un nivel de educación básico, mientras que el estudio nacional se realizó con una población aleatoria en la que no todos los integrantes de la muestra tenían un nivel de escolaridad técnico. Cabe anotar que estudios nacionales anteriores han mostrado relevancias positivas en las personas que tienen un mayor grado de escolaridad y su salud dental [6].

Esta diferencia de prevalencias también se evidenció al compararla con el estudio realizado por Aguilar [19] en la Universidad Autónoma de Nayarit, y el de Castillo [20] en la Universidad de Cuenca (Ecuador-2014), cuyos resultados de prevalencia de caries fueron de $68,9 \%$ y $89 \%$, respectivamente. Es importante resaltar que estos estudios se realizaron en estudiantes de odontología en etapa de formación más avanzada que los evaluados en el SENA.

En cuanto a la valoración de la experiencia de caries dental, se encontró que 67,7\% de los aprendices tiene o ha tenido en algún momento de su vida caries dental. Dando continuidad al análisis de estos resultados con elúltimo Estudio Nacionalde Salud Bucal, resulta menor la experiencia de caries de los aprendices del SENA, dado que en los adultos jóvenes entre 20 y 34 años evaluados en el ENSAB IV [21] la experiencia de caries se reporta en $87,96 \%$. Una consideración importante al momento de relacionar estos resultados es que la muestra evaluada en el ENSAB fue mayor que la del SENA.

Al evaluar estos resultados con la prevalencia de caries valorada en los estudiantes de la Universidad Veracruzana de México, donde fue de $80,9 \%$, continúa siendo menor la prevalencia de los estudiantes del programa técnico del SENA [22].

Se encontró que el promedio de dientes cariados por individuo examinado es de 0,42 , es decir, que hay menos de un diente cariado en los aprendices valorados. Este dato es positivo si se compara con los resultados de otro estudio realizado en estudiantes del área de la salud bucal, en el que el promedio de dientes cariados fue de 2,9 [19] e incluso es menor que en el ENSAB IV, donde el promedio fue de 1,47 [21].

\section{Conclusión}

Diferentes fuentes han demostrado que pese a la existencia de algunas desigualdades, los índices de caries (prevalencia, experiencia) han mejorado [6], [23]. Si bien en este estudio no hubo asociaciones estadísticamente significativas, la mejoría en los índices al compararse con algunos referentes puede obedecer a actitudes individuales, en particular conocimientos, hábitos y prácticas que están relacionadas con el uso masivo de las cremas dentales fluoradas, el consumo de agua con flúor, el uso correcto de los elementos de higiene oral, como ha sido reportado en otras investigaciones a nivel mundial, así como a la implementación de programas preventivos masivos.

\section{Referencias}

[1] Alcaldía Mayor de Bogotá. Guías de práctica clínica en salud oral. 2011. Disponible en: http://www. saludcapital.gov.co/SUBS/Documents/Guias\%20 2011.pdf

[2] Revista Médica Electrónica. Comportamiento de la caries dental en escolares obesos y normopesos de 8 a 13 años. 2010. Disponible en: http:// scielo.sld.cu/scielo.php?script=sci_arttext\&pi$\mathrm{d}=$ S1684-18242010000300002\&lng=es.

[3] Toscón J, Cabrera G. Algunas creencias sobre susceptibilidad y severidad de la caries en adolescen- 
tes del Valle del Cauca, Colombia. Colomb Med. 2005;36:140-5.

[4] Olmos P, Piovesan S, Musto M, Lorenzo S, Álvarez $\mathrm{R}$, Massa F. Caries dental. La enfermedad oral más prevalente: primer estudio poblacional en jóvenes y adultos uruguayos del interior del país. Odontoestomatol. 2013;15(n.esp):26-34.

[5] Acevedo L, Monroy Y. Impacto que ha tenido la medida de promoción y prevención en salud oral en los últimos cinco años de la población correspondiente al régimen subsidiado de la ESE empresa de salud del municipio de Soacha. Bogotá: Universidad EAN; 2013.

[6] Asociación Colombiana de Facultades de Odontología (ACFO). Resultados del III Estudio Nacional de Salud Bucal. 2013. Disponible en: http://acfo.edu.co/ investigacion/index.php/publicaciones-en-linea?i$\mathrm{d}=28$ :resultados-del-iii-estudio-nacional-de-salud-bucal\&catid=20:en-linea\&\%20(acfo)

[7] Jiménez S, Medina C, Lara E, Scougal R, De la Rosa $\mathrm{R}$, Márquez $\mathrm{S}$, et al. Desigualdades socioeconómicas en la utilización de servicios de salud bucal (USSB) alguna vez en la vida por escolares mexicanos de 6-12 años de edad. Gac Med Mex. 2015;(151):27-33.

[8] Márquez M, Rodríguez R, Rodríguez Y, Estrada G, Aroche A. Epidemiología de la caries dental en niños de 6-12 años en la Clínica Odontológica La Democracia. Medisan. 2009;13(5).

[9] Alejandro Ramírez Peña. Si una persona recibe más de $\$ 211.807$ mensuales ya supera la pobreza. El Tiempo. 25 de marzo de 2015.

[10] Álvarez MJ. Desigualdades en Colombia. 2013. Disponible en: http://journals.iai.spk-berlin.de/index. php/iberoamericana/article/viewFile/381/65

[11] Servicio Nacional de Aprendizaje (sENA). Quiénes somos. Disponible en: http://www.sena.edu. co/acerca-del-sena/quienes-somos/Paginas/Quienes-Somos.aspx

[12] Servicio Nacional de Aprendizaje (sENA). Objetivos y funciones. Disponible en: http://www.sena.edu. co/acerca-del-sena/quienes-somos/Paginas/Objetivos-y-Funciones.aspx

[13] Organización Mundial de la Salud (oms). Encuesta de salud bucodental. Métodos básicos. 4. ${ }^{a}$ ed. Ginebra: OMs; 1997. Disponible en: http://whqlibdoc. who.int/publications/1997/9243544934_spa.pdf

[14] República de Colombia, Ministerio de Salud. Resolución 003430, Por la cual se establecen las normas científicas, técnicas y administrativas para la investigación en salud (4 oct 1993). Disponible en: http:// www.unisabana.edu.co/fileadmin/Documentos/Investigacion/comite_de_etica/Res_8430_1993_-Salud.pdf

[15] Zelocuathecatl A, Sosa N, Ortega M, De la Fuente J. Experiencia de caries dental e higiene bucal en escolares de una comunidad indígena del estado de Oaxaca. Rev Odont Mex. 2010;14(1):32-7. Disponible en: http://www.medigraphic.com/pdfs/odon/ uo-2010/uo101e.pdf

[16] Martínez MC, Morales S, Martínez CM. Caries dental en adultos jóvenes en relación con características microbiológicas y fisicoquímicas de la saliva. 2013;15(6):867-77. Disponible en: http://www. redalyc.org/articulo.oa?id $=42231751007$

[17] Kohli A, Poletto L, Pezzotto S. Hábitos alimentarios y experiencia de caries en adultos jóvenes en Rosario, Argentina. Archivos Latinoamericanos de Nutrición. 2007;57(4).

[18] Solano JC, Castaño A. García LV. Tendencias actuales en el personal auxiliar de odontología. Resultados de una aproximación multivariante. Arch Odont Estomat. 2000;16(1):13-21.

[19] Aguilar N, Navarrete K, Robles D, Aguilar S, Rojas A. Dientes sanos, cariados, perdidos y obturados en los estudiantes de la Unidad Académica de Odontología de la Universidad Autónoma de Nayarit. Rev Odontol Latin. 2009;1(2):27-32.

[20] Castillo R. Situación de la salud bucal según prevalencia de caries, higiene oral y patologías orales más frecuentes de los estudiantes de odontología de la Universidad de Cuenca, 2014. Universidad de Cuenca; 2015. p. 1-49.

[21] Pontificia Universidad Javeriana, Ministerio de Salud. IV Estudio Nacional de Salud Bucal ensab IV 2013-2014. Bogotá: Ministerio de Salud; 2015. p. 378.

[22] Gómez N, Morales M. Determinación de los Índices CPO-D e IHOS en estudiantes de la Universidad Veracruzana, México. Rev Chil Sal Públ. 2012;16(1)2631. Disponible en: http://www.revistasaludpublica. uchile.cl/index.php/RCSP/article/view/18609

[23] Montero M, Rojas F, Socorro M, Torres J, Acevedo A. Experiencia de caries y fluorosis dental en escolares que consumen agua con diferentes concentraciones de fluoruro en Maiquetía, Estado Vargas, Venezuela. Invest Clin. 2007;48(1):5-19. 